\title{
Update on Medical Treatment of Patients With Eisenmenger Syndrome
}

\author{
Hiroyuki Fukushima, ${ }^{1}$ MD
}

\begin{abstract}
SUMMARY
Eisenmenger syndrome (ES) is the most advanced form of pulmonary arterial hypertension (PAH) associated with congenital heart defects. ES greatly affects functional capacity, quality of life, and survival of patients. Clinical management of patients with ES has been dramatically changed and developed in a new therapeutic era of PAH, approximately a century after the first ES case described by Dr. Eisenmenger. Targeted drugs for PAH improve functional capacity and quality of life of patients with ES. In addition, these drugs may also provide a benefit for patients' survival. To achieve further development in medical treatment of patients with ES, they will need to be individualized according to functional classes, underlying cardiac defects, and underlying systemic diseases. (Int Heart J 2015; 56: S4-S7)
\end{abstract}

Key words: Pulmonary arterial hypertension, Congenital heart defects, Advanced targeted therapy

$\mathrm{T}$ his article updates the medical treatment of patients with Eisenmenger syndrome (ES) focusing on current evidence and future perspectives in a new therapeutic era of pulmonary arterial hypertension (PAH). The gist of this article was presented at the PAH-Coronary Heart Disease Expert Board in Japan held in Kobe in July 2011.

Clinical management of patients with ES has traditionally focused on palliative and supportive treatment. However, increased understanding of the pathophysiology of ES and development of disease-specific, targeted therapies for idiopathic or collagen diseases associated with PAH have provided an important challenge in management of ES.

\section{Chronology of ES}

Figure 1 shows the chronology of ES. There were four important episodes in the history of ES, including the first report of a case by Dr. Eisenmenger in 1897, ${ }^{1)}$ the establishment of pathophysiology by Dr. Wood in $1958,{ }^{2)}$ the first therapeutic evidence by the Bosentan Randomized Trial of Endothelin Antagonist Therapy-5 (BREATHE-5) study in 2006, ${ }^{3)}$ and an important report suggesting improved survival of ES with PAHtargeted therapy in $2010 .{ }^{4}$

The first reported case of Eisenmenger syndrome was a 32-year-old man with a large ventricular septal defect (VSD), overriding the aorta and cyanosis. He died because of hemoptysis. ${ }^{1)}$ Dr. Eisenmenger reported his clinical course and necropsy findings. This report was the start of the challenge in understanding ES.

About half a century after the first report, Dr. Wood published an important manuscript. ${ }^{2}$ He summarized the clinical and physiological features of 127 cases and established the definition of ES. He also precisely described each case of Ei- senmenger syndrome, including concrete data. Even in the modern era, his case reports still provide understanding of the essence of ES.

Approximately another 50 years after Dr. Wood's report, the first therapeutic evidence of ES was achieved by the BREATHE-5 study in 2006. ${ }^{3)}$ This study showed that bosentan, one of the targeted therapies for PAH, was beneficial for functional capacity in patients with ES. This was a groundbreaking study in the medical treatment of ES. In addition, advanced targeted therapy improved survival of patients with ES in 2010. ${ }^{4)}$ This result gave new hope to ES patients.

\section{Treatment of ES in a New Era}

Historically, management options for patients with ES have been limited to a palliative fashion or heart-lung transplantation. Medical treatment for ES often includes the use of diuretics, digoxin, antiarrhythmics, and anticoagulants. However, none of these approaches has proved to significantly modify the quality of life, survival, or risk of deterioration in patients with ES. Currently, disease-specific, targeted PAH treatment has been applied to ES and has been shown to improve patients' functional capacity and quality of life.

Endothelin receptor antagonists: The BREATHE- 5 trial ${ }^{3)}$ first showed that bosentan improved the exercise capacity of ES patients in World Health Organization (WHO) functional class 3 . To date, the most efficacious targeted PAH therapy in patients with ES is bosentan. The BREATHE-5 trial was the first double-blind placebo-controlled trial in patients with ES. In the BREATHE- 5 trial, patients received bosentan $125 \mathrm{mg} / \mathrm{day}$ or placebo for 4 weeks, and then bosentan $250 \mathrm{mg} /$ day or placebo for the remaining 12 weeks. Although the BREATHE-5 trial was only 16 weeks in duration, which is a relatively short

From the ${ }^{1}$ Department of Pediatrics, Keio University School of Medicine, Tokyo, Japan.

Address for correspondence: Hiroyuki Fukushima, MD, Department of Pediatrics, Keio University School of Medicine, 35 Shinanomachi, Shinjuku-ku, Tokyo 1608582, Japan. E-mail: fuku-h@z7.keio.jp

Received for publication October 28, 2014. Accepted October 28, 2014

Relesed online on J-STAGE March 19, 2015.

All rights reserved by the International Heart Journal Association. 
1897 First report by Victor Eisenmenger

1958 Establishment by Paul Wood

2006 First therapeutic evidence: BREATHE-5

\section{Improved survival with targeted therapy}

Figure 1. Chronology of ES.

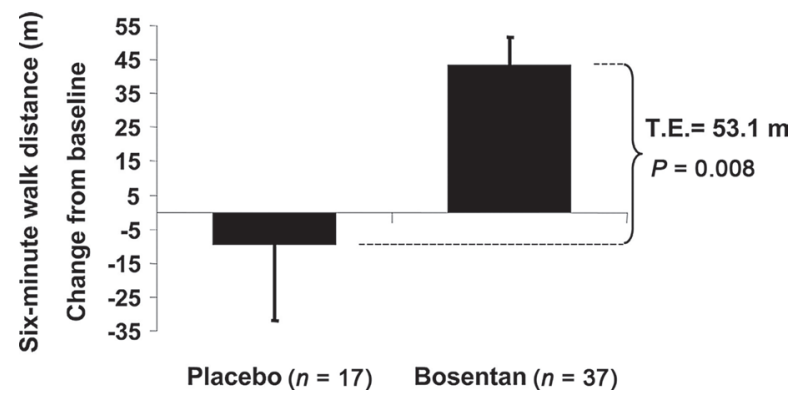

Figure 2. Graph showing the change from baseline in 6-minute walking distance to after treatment with bosentan or placebo. This distance was significantly improved in the bosentan group compared with the placebo group. TE indicates treatment effect. Reproduced with permission from Galiè N, et al. Circulation 2006; 114: 48-54. ${ }^{3)}$

period of time for patients with ES whose natural history is more protracted than those with idiopathic PAH, bosentan improved the exercise capacity of patients with ES. Figure 2 shows the change from baseline of 6-minute walking distance in the placebo and bosentan groups. The distance decreased in the placebo group by $9.7 \mathrm{~m}$, but increased in the bosentantreated group by $43.4 \mathrm{~m}$. Consequently, bosentan showed a treatment effect of $53.1 \mathrm{~m}$.

In addition, an open-label extended trial ${ }^{5)}$ showed that improved exercise capacity observed in the BREATHE- 5 trial was maintained after 24 additional weeks of the bosentan administration period. Figure 3 shows the change in 6-minute walking distance from baseline of the BREATHE- 5 trial to the end of the BREATHE-5 open-label extended trial. In the exbosentan group, which received bosentan in the BREATHE- 5 trial, an increased distance was maintained at the end of the open-label extended study. In the ex-placebo group, which received placebo in the BREATHE-5 trial, the 6-minute walking distance was increased by 24 weeks of bosentan administration. These data implicate bosentan as a new evidence-based therapeutic option for patients with ES.

Phosphodiesterase type-5 inhibitors: An open-label uncontrolled study reported that 6 months of sildenafil administration improved the New York Heart Association (NYHA) functional class and systemic arterial oxygen saturation in patients with ES. However, the 6-minute walking distance showed no significant change. ${ }^{6}$ Another prospective study showed that 3 months of oral sildenafil therapy improved the quality of life, which was assessed using a disease-specific questionnaire in

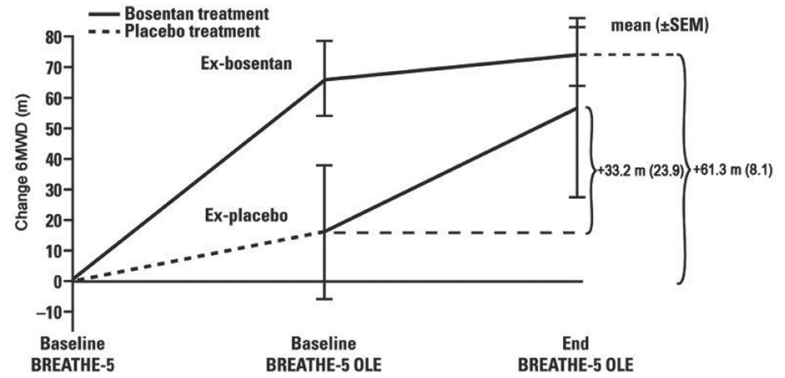

Figure 3. Graph showing the change in 6-minute walking distance from baseline of BREATHE-5 to the end of BREATHE-5 (week 16) and after 24 weeks of bosentan in the open-label extension (OLE) (week 40) for explacebo patients and ex-bosentan patients. An improvement in distance was achieved at week 40 in both groups. Reproduced with permission from Gatzoulis MA, et al. Int J Cardiol 2008; 127; 27-32.

addition to 6-minute walking distance and WHO functional class. $^{7)}$

Prostacyclin and prostacyclin analogs: A retrospective review on 8 patients with ES showed that 3 months of epoprostenol administration resulted in improved WHO functional class, increased oxygen saturation, decreased pulmonary vascular resistance, and increased 6-minute walking distance. ${ }^{8)}$ However, epoprostenol therapy also has problems concerning long-term catheter use (eg, catheter-related infections and thrombi).

\section{Effect of PAH-Targeted Therapy on Survival in ES Patients}

Advanced targeted therapy for PAH had been shown to improve exercise capacity, pulmonary hemodynamics, and quality of life in patients with ES. However, until recently, no data existed on a potential survival benefit. Four years after the first therapeutic evidence by BREATHE-5, an important study was published suggesting that advanced and targeted therapy for PAH improves survival of patients with ES. ${ }^{4)}$ This was a retrospective study from a single pulmonary hypertension center. A total of 229 adult patients with ES were included. Approximately half of them had complex anatomy and 53.7\% were WHO class 3 or 4 . The mean resting oxygen saturation was $84.3 \%$. Sixty-eight patients $(29.7 \%)$ were on advanced targeted therapy. Ten of them were on advanced therapy at the time of first assessment and 58 were started on advanced therapy during follow-up. In this study, advanced therapy was started on bosentan (73.5\%), sildenafil (25.0\%), or epoprostenol (1.5\%). Fifty-two of 229 patients died during a median follow-up of 4.0 years. The overall 5-year mortality rate was $23.3 \%$. Adjusted survival rate curves with and without advanced therapy are shown in Figure 4. The mortality rate of patients with advanced therapy was much lower than that of patients without advanced therapy.

A retrospective study of adult patients with ES in Japan also suggested that advanced targeted therapy improves survival rate in patients with ES (Figure 5). ${ }^{9}$

\section{Treatment Algorithm for ES}

Figure 6 shows one of the current treatment algorithms for ES. In this algorithm, advanced targeted therapies are recommended for patients in NYHA classes 3 and 4. Dimopoulos, et $a l^{4)}$ and Sakazaki, et $a l^{9)}$ supported the accuracy of this algorithm from the viewpoint of survival benefit. However, 


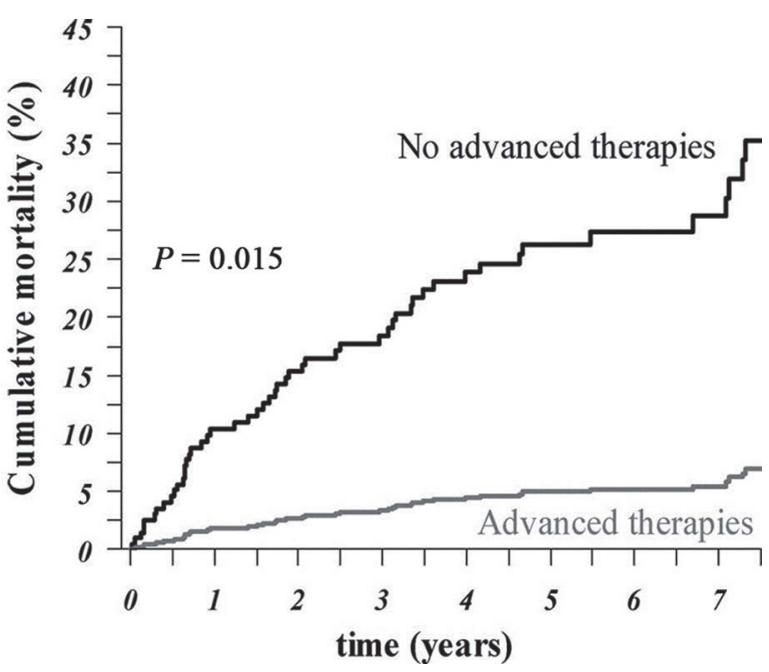

Figure 4. Graph showing adjusted survival rate curves, based on the propensity score-adjusted Cox model, of patients within the third propensity score quartile with and without advanced therapy. Reproduced with permission from Dimopoulos K, et al. Circulation 2010; 121: 20-5. ${ }^{4)}$

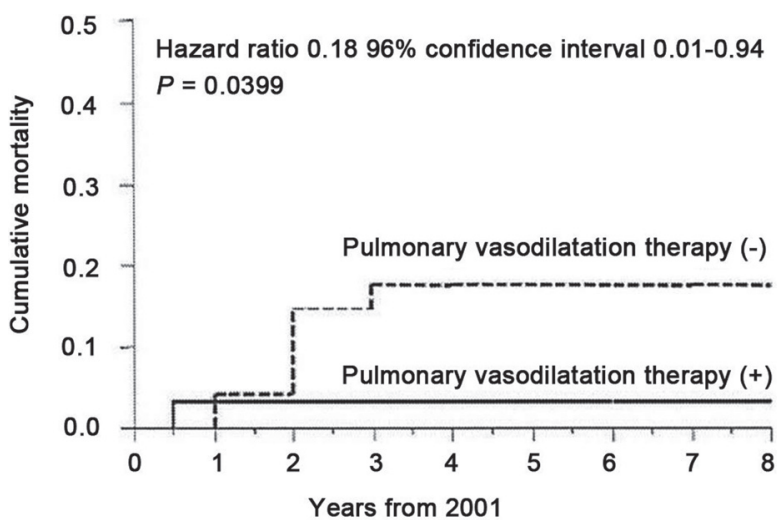

Figure 5. Graph showing adjusted cumulative mortality rate curves, based on the propensity score-adjusted Cox model, of patients with and without advanced therapy. Reproduced with permission from Sakazaki H, et al. Pediatr Cardiol Card Surg 2011; 27: 121-31.'

several uncertain issues remain (eg, treatment strategy for patients in NYHA class 1 or 2).

\section{Future Perspectives}

Individualization is an important concept concerning future perspectives in treatments for patients with ES. Recently, medical treatment has been developed by consolidation of several types of pulmonary hypertension, including ES into PAH. However, further development will require individualization by functional classes, underlying cardiac anomalies, and underlying systemic diseases.

Dimopoulos, et $a l^{4)}$ showed that the 5-year survival rate in patients in NYHA class 1 or 2 was $14.1 \%$. This appears to be higher than that in patients with advanced therapy. Nevertheless, the functional class of a vast majority of patients with advanced therapy was NYHA class 3 or 4 at baseline. Therefore, use of advanced therapy for ES patients in NYHA class 2

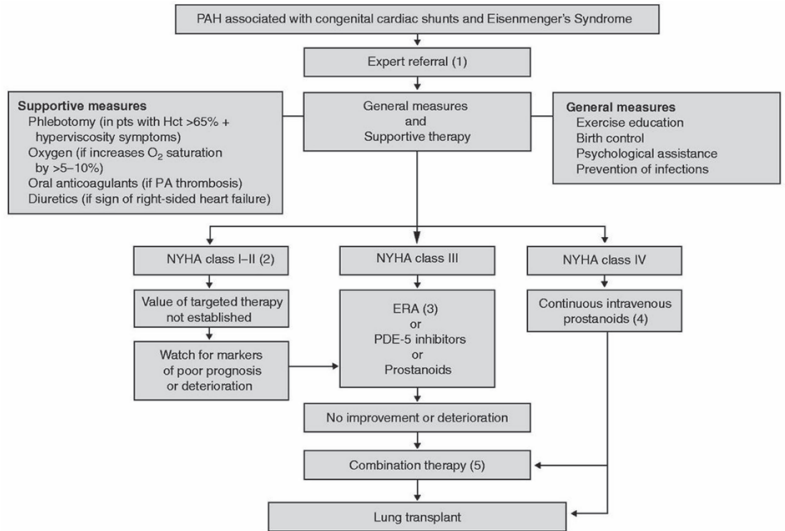

Figure 6. Treatment algorithm for patients with PAH associated with congenital cardiac shunts and Eisenmenger syndrome. Hct indicates hematocrit; PA, pulmonary artery; and PDE-5, phosphodiesterase-5. Reproduced with permission from Galie N, et al. Drugs 2008; 68: 1049-66. ${ }^{(0)}$

(and 1) may be reasonable from the viewpoint of survival benefit.

Tailor-made advanced therapy according to underlying cardiac anomalies (eg, atrial septal defect [ASD] and VSD) is also important. A subgroup analysis of BREATHE- $5^{11)} \mathrm{did}$ not show any difference in the effect of bosentan between patients with ASD and VSD. Nonetheless, there are several differences between ASD and VSD in ES patients from the viewpoint of rapidity and severity of pulmonary vascular occlusive disease, the timing of diagnosis of defects, and hemodynamics, including volume load of both ventricles. Therefore, further studies are required to investigate the similarities and differences in the efficacy of advanced therapy between ES patients with ASD and those with VSD.

Underlying systemic diseases, such as Down syndrome or polysplenia, may also affect advanced therapy. Down syndrome was present in approximately $30 \%$ of patients in the report by Dimopoulos, et ll $^{4)}$ However, there is no description of the difference between Down and non-Down patients. An imbalance in biosynthesis of thromboxane $\mathrm{A}_{2}$ and prostacyclin has been reported in Down syndrome. ${ }^{12)}$ Findings concerning factors contributing to the pathogenesis of PAH will provide tailor-made advanced targeted therapy for individual ES patients according to underlying systemic diseases.

Conclusion: In a new therapeutic era for PAH, approximately a century after the first case report of ES by Dr. Eisenmenger, medical treatment for patients with ES has considerably developed. Advanced, disease-specific, targeted therapy (eg, endothelin receptor antagonists, phpsphodiesterase-5 inhibitors, prostacyclin, and prostacyclin analogues) will improve ES patients' functional capacity and quality of life. Moreover, these drugs may provide a benefit for survival.

To achieve further development in the treatment of ES, individualization of ES patients is essential. Dr. Wood, who passionately cared for many ES patients and precisely described their clinical and pathological features, made an important contribution to understanding a variety of pathogeneses of ES. His article is a reminder of the value of case reports, as well as clinical evidence, achieved by a large amount of patients. We need to provide the best therapeutic option to indi- 
vidual patients with ES, describe their clinical course, share this information with patients and doctors worldwide, and establish a concrete and individualized (tailor-made) guideline of medical therapy for patients with ES in a new therapeutic era of PAH.

\section{ACKNOWLEDGMENTS}

The authors would like to thank Springer Healthcare and Ellen Knapp, $\mathrm{PhD}$, for providing English-language editing, which was funded by Actelion Pharmaceuticals Japan Ltd.

\section{REFERENCES}

1. Eisenmenger V. Die angeborenen Defecte der Kammerscheidewand des Herzens. Z Klin Med 1897; 32: 1-28. (German)

2. Wood P. The Eisenmenger syndrome or pulmonary hypertension with reversed central shunt. Br Med J 1958; 2: 701-9.

3. Galiè N, Beghetti M, Gatzoulis MA, et al. Bosentan therapy in patients with Eisenmenger syndrome: a multicenter, double-blind, randomized, placebo-controlled study. Circulation 2006; 114: 4854.

4. Dimopoulos K, Inuzuka R, Goletto S, et al. Improved survival among patients with Eisenmenger syndrome receiving advanced therapy for pulmonary arterial hypertension. Circulation 2010; 121: 20-5.
5. Gatzoulis MA, Beghetti M, Galiè N, et al; BREATHE-5 Investigators. Longer-term bosentan therapy improves functional capacity in Eisenmenger syndrome: results of the BREATHE-5 open-label extension study. Int J Cardiol 2008; 127: 27-32.

6. Chau EM, Fan KY, Chow WH. Effects of chronic sildenafil in patients with Eisenmenger syndrome versus idiopathic pulmonary arterial hypertension. Int J Cardiol 2007; 120: 301-5.

7. Tay EL, Papaphylactou M, Diller GP, et al. Quality of life and functional capacity can be improved in patients with Eisenmenger syndrome with oral sildenafil therapy. Int J Cardiol 2011; 149: 372-6.

8. Fernandes SM, Newburger JW, Lang P, et al. Usefulness of epoprostenol therapy in the severely ill adolescent/adult with Eisenmenger physiology. Am J Cardiol 2003; 91: 632-5.

9. Sakazaki H, Niwa K, Ueno M, et al. Clinical study of adult patients with Eisenmenger syndrome in Japan. Pediatr Cardiol Card Surg 2011; 27: 121-31.

10. Galie N, Manes A, Palazzini M, et al. Management of pulmonary arterial hypertension associated with congenital systemic-to-pulmonary shunts and Eisenmenger's syndrome. Drugs 2008; 68: 1049-66. (Review)

11. Berger RM, Beghetti M, Galiè N, et al. Atrial septal defects versus ventricular septal defects in BREATHE-5, a placebo-controlled study of pulmonary arterial hypertension related to Eisenmenger's syndrome: a subgroup analysis. Int J Cardiol 2010; 144: 373-8.

12. Fukushima H, Kosaki K, Sato R, et al. Mechanisms underlying early development of pulmonary vascular obstructive disease in Down syndrome: An imbalance in biosynthesis of thromboxane A2 and prostacyclin. Am J Med Genet A 2010; 152A: 1919-24. 